\title{
A City Guide Agent Creating and Adapting Individual Sightseeing Tours Based on Field Trial Results
}

\author{
Ronny Kramer, Marko Modsching and Klaus ten Hagen \\ University of Applied Sciences Zittau/Görlitz \\ Brückenstr.1, D-02826 Görlitz, Germany \\ \{RKramer, Mmodsching, K.tenHagen\}@HS-ZiGr.de
}

\begin{abstract}
Tourists deciding to explore a destination spontaneously on their own often end up following the majority to the crowded locations. Today's agent technology provides the opportunity for intelligent guides taking care of the whole tour organisation and execution in time and thus providing individual experiences. This is the main objective of the Dynamic Tour Guide (DTG) - a mobile agent that selects attractions, plans individual tours, provides navigational guidance and offers location based interpretation. Over all it consistently adapts the tour to a tourist's specific behaviour in order to provide any possible support via a mobile device. A field trial served to clarify fundamental questions to achieve personality in mobile context: Is it possible to seed interest profiles that allow the accurate prediction of actual rankings of sights? Are these profiles sufficiently diverse to base personalised tours on individual interests? Do personal tours really affect spatial behaviour of tourists? Analyzing the captured interest profiles gives an insight into their actual diversity, discusses their necessity and helps simulating an improved distribution of tourists at a destination.
\end{abstract}

Keywords: Dynamic Tour Guide, Semantic Matching, Preference Elicitation, Tour Calculation, Spatial Behaviour.

\section{Introduction}

Presently available guided tours need to be booked in advance. Tourists wanting to start a tour on the spot will have to assemble their own one using public information sources like maps or signs. With just minimal information a software agent might take on that challenge. A tourist needs an interest profile, a start- and end point and a given time period - all necessary data to compute a tour. This is described by their personal context which needs to be mapped with the environmental context (e.g. available sights and services) at the destination. Context means all available information at a certain location for a certain time related to a certain entity. The challenge is to compute an optimal tour given the personal and local context whereas the tourist can modify the proposed tour afterwards.

During the execution of the tour the tourist will be guided to the next Tour Building Block (TBB, which may be a sight or restaurant) using standard navigation software, like MS Mappoint or Navigon [21]. When the tourist starts walking the DTG determines the actual walking speed of the tourist on this day given the conditions of the sidewalks and streets. This update of the personal context might make a recalculation of the remaining tour necessary. As soon as a tourist approaches a point where a TBB becomes visible she/he will be provided introductory information via a headset suitable to the direction from which the tourist is approaching the TBB. Otherwise the tourist might soon get disorientated when approaching from e.g. the opposite side. As long as she/he is in the proximity of the TBB, the tourist will receive audio information. Some tourists will decide to explore the TBB further by e.g. walking into the court yard. In this case additional information appropriate to current context is provided. As soon as she/he leaves the TBB, the information provision will be stopped and the navigational guidance towards the next TBB is restarted. In case the tourist stays much longer than initially assumed, the tour will have to be recalculated for the remaining amount of time.

On the way to the next TBB some tourists might get distracted by another attraction be it another sight or simply a shop. Then the DTG will interrupt the navigational hints and provide information for the current context if available. In case of a spontaneous visit to a local store the DTG will simply wait for the tourist to leave the location to continue on a tour recalculated for the remaining amount of time. Despite the navigational guidance through audio hints some tourists might get on the wrong path. The navigation software will try to get the tourist back on a path towards the next TBB. However beyond a certain deviation it becomes more meaningful to adapt the sequence of TBBs to the new location of the tourist. 
The concrete implementation of these scenarios is described in more detail in the first part of this article. The second part deals with a field study executed in Görlitz and aimed to analyze the current distribution and behavior of tourists. Furthermore three methods for eliciting ones preferences were designed and examined in regard of their applicability. The specification of interests in mobile context is difficult as a mobile device provides less than $4 \%$ of the pixels of a PC, many distractions, e.g. traffic noise, make tourists less patient in interacting with an application. Thus time and information bandwidth is severely limited compared to a standard PC environment. The gathered interest profiles were subsequently benchmarked in order to determine the accuracy of the elicitation process. The diversity of the tourists' interests were closely analyzed in order to study the necessity to gather individual profiles. A simulation of tours based on the gathered profiles indicates that an improvement of the tourists' spatial distribution can actually be achieved by adopting a mobile agent like the DTG.

\section{Related work and challenges}

In a survey of tourists in Heidelberg by Freytag [10] around 1500 tourists were asked about their activities during their visit of the city in 2003. The first important fact to mention is that most tourists explore the city by foot and on their own. Only $7 \%$ decide for a guided tour. The second finding indicates that most tourists move within a very limited area around the Old Town. Almost anybody visits the castle while all other sights receive less attention; some even less than $5 \%$. This implies that most tourists gather at a few places. This is probably an effect of missing contextual information. One can predict that, provided better information, e.g. via a mobile device by the DTG, more tourists would visit attractions appropriate to their interests. In chapter 9 of Kempermann et al [16] an examination of the different behavior of first-time and repeat tourists at theme park destinations is presented. It is outlined that first-time visitors have less information about a destination and try to visit as many attractions as possible, whereas repeat visitors select the attractions they attend more properly because they already know what to expect. The DTG may help tourists visiting destinations for the first time to be able to pick out the sights they are most interested in from the start by providing all information that are invisible or inaccessible for tourists.

But Tour Guide Agents aren't a new invention. There have been numerous research activities in this sector over the past few years. The following projects summarize the current state of the art:

- GUIDE [3] is a mobile tour guide which concepts are most related to the DTG. The visitor chooses attractions from various categories. These attractions are then sequenced by the GUIDE taking into account opening hours, best time to visit and the distance between attractions. The sequence can be modified manually. Navigation is achieved by a map and a list of instructions. Reaching a point of interest (POI), context-sensitive information is provided. Differences are the use of cell based positioning instead of GPS and the selection of concrete sights to visit by the tourist him/herself instead of using generic preferences to compute a ranking which then drives the computation of the initial tour plan. The DTG also adapts to the actual behavior of the tourist by recomputing the tour for the remaining amount of time. Lacking a ranking the GUIDE re-arranges the sequence of initially selected attractions.

- Cyberguide [1] was one of the first mobile tour guides. It works outdoor with GPS and indoor with infrared to determine context information like users' position and orientation. Personal preferences are not analyzed to compute a tour plan, but the user can receive information about anything he/she sees, wherever he/she is. Requesting a route to a desired POI is possible too. In addition it provides the option to create a kind of diary about the whole tour.

- The Crumpet project [4] enables a mobile agent to find certain sights, to present them on a map and to calculate a route to a selected one.

- The software developed by eNarro [8] provides predetermined tours presenting the most important sights in many big cities all over the world. The tourist needs a PDA with a special player loaded with the content for the particular tour. She/he also has to have navigation software which will lead her/him to the different places. The attractions are then presented using audiovisual information.

- In connection with the AgentCities [2] framework the "Fujitsu Laboratories of America" [11] have developed an event organizer. Based on an ontology, it selects a restaurant according to the guest's preferences and makes a reservation when planning an evening. This is a step towards context-awareness, because the search for a restaurant is dynamic due to the user's preferences.

In contrast to existing tour guides the DTG computes an individual tour in real-time by considering available context information like personal interests and location based services. In order to build the DTG the following challenges have to be addressed:

1. Elicitation of generic interests of a tourist in a mobile context to seed the profile

2. Ranking of TBBs by semantic matching

3. Computation of a tour in less than 5 seconds

4. Representation of context based information

5. Tour tracking and adaptation

Eliciting the tourists' interests is the precondition to be able to make any recommendations. Websites often use collaborative browsing [22]. In this case the application is 
hosted by a central server and thus aggregating and analyzing the browsing behavior of all users is natural.

Fink and Kobsa [26] investigated the elicitation of preferences in order to personalize a tour. They also propose to observe the behavior of the tourist, generalize the observations based on "stereotypes" and then predict interests in certain concrete items. This approach presumes a central server to aggregate and mine observations. In order to bootstrap this bottom-up recommender a certain amount of observations needs to be gathered prior to derive stereotypes and then recommendations. The DTG approach intends to develop and validate methods to gather a personal interest profile. This generic interest profile is then used to derive rankings on concrete TBBs by semantic matching based on a common public ontology of the destination. The top-down DTG recommender gathers personal data using a mobile device owned by the user. Importantly, personal information does not leave the boundary of the personal computing device.

Gretzel and Fesenmaier [14] not only mention the recommendation aspect, but also emphasize the persuasive component of a recommendation system. The primary goal is to get to know the preferences of users, but as this is a complex task it is better to get some clues and then to suggest things what can influence the choice of users by the way of representation. Most people aren't aware about their preferences all the time, so they need to be inspired to be reminded of it.

Eliciting ones preferences is not a trivial task and there is no general solution to do so at least not in mobile context. But solving this problem may lead to fundamental improvements in eTourism, e.g. more personalized information provision to enable the tourists to enjoy a destination to its full potential, which shall manifest itself by dispersion over a much wider area of the city.

\section{The Dynamic Tour Guide}

\section{A. Architecture}

Each sight, as a possible component of the tour $(\mathrm{TBB}=$ Tour Building Block), is semantically modelled by a content provider using the DTG AuthoringTool. Each TBB will have its own web service (WS). A service provider like a restaurant will wrap the local restaurant management system by a WS. This WS will provide the semantic model, current information, e.g. opening hours, and a transactional interface to e.g. reserve a table. The WSs of the TBBs are published at a UDDI registry.

The DTG server is executing a semantic match algorithm to rank the sights for a specific tourist. A computationally more demanding task of the DTG server is the computation of a tour as a sequence of TBBs. Audio hints and a map for navigation are provided by standard navigation software to guide the tourist to the next TBB. The DTG provides information about a TBB as the tourist approaches it. Furthermore it adapts the higher-level plan for the remaining time to the actual walking speed and staying time at a TBB. Expectedly most people will own a mobile device in the next couple of years, cities will be covered with WLAN access points and DGPS will provide localization with a precision of at least $1 \mathrm{~m} \mathrm{[5].} \mathrm{This} \mathrm{enables} \mathrm{the} \mathrm{following} \mathrm{features} \mathrm{and}$ interactions:

- Localisation:

The mobile device is aware of its current position, either in a city via e.g. the Global Positioning System (GPS-WAAS) or inside buildings like museums via WLAN, Infrared grids or RFIDs.

\section{- Service discovery:}

After arrival at a destination the DTG will determine the next DTG server in a UDDI registry. Based on the personal context like the maintained interest profile and the time period set by the tourist, the DTG will discover the local context like sights and services at this destination by scanning another UDDI registry, interrogate the corresponding web services to update the current information and then compute potential tours.

- Navigation and tour adaptation:

After the tourist has selected and optionally modified a tour, the local navigation software will visualize the tour on a map and guide the tourist via audio information. In the background the DTG will consistently track the execution of the ongoing tour for contextual changes, e.g. any deviations like changing walking speeds or additional breaks, by recalculating the tour to make sure that the tourist arrives at the desired endpoint in time.

\section{B. Tour composition}

Semantic matching is applied to select TBBs according to the personal interests of a tourist. This task is different for any tourist as the contexts always differ. The personal context of the tourist has to be mapped with the local one. The interests, the available time period and the position of the tourist are most important. Based on this information a human expert can decide which tour would possibly fit best, but the challenge is to let the decision be made by a program. Therefore the computer needs to understand the meaning of certain data. The solution is to define a common knowledge base, containing all possible terms, arranging relations like synonyms and defining attributes - an ontology. It's a model of a specific area of reality. Every concept, existing in the real world, is displayed as a class. Relations between classes result in a hierarchical structure of all concepts, where each class can have parent classes and child classes. Attributes serve to define properties in order to describe classes more precisely.

The ontology is used to semantically model the interests of the tourist and to classify the TBBs what means that profiles are generated containing the associated classes of the ontology. All existing sights of a city (here: Görlitz [13]) 
need to be assigned to main categories of interests, which have little in common. For Görlitz these were defined as: architecture, celebrities, culture and leisure, landmarks and landscape, shown in Figure 2.

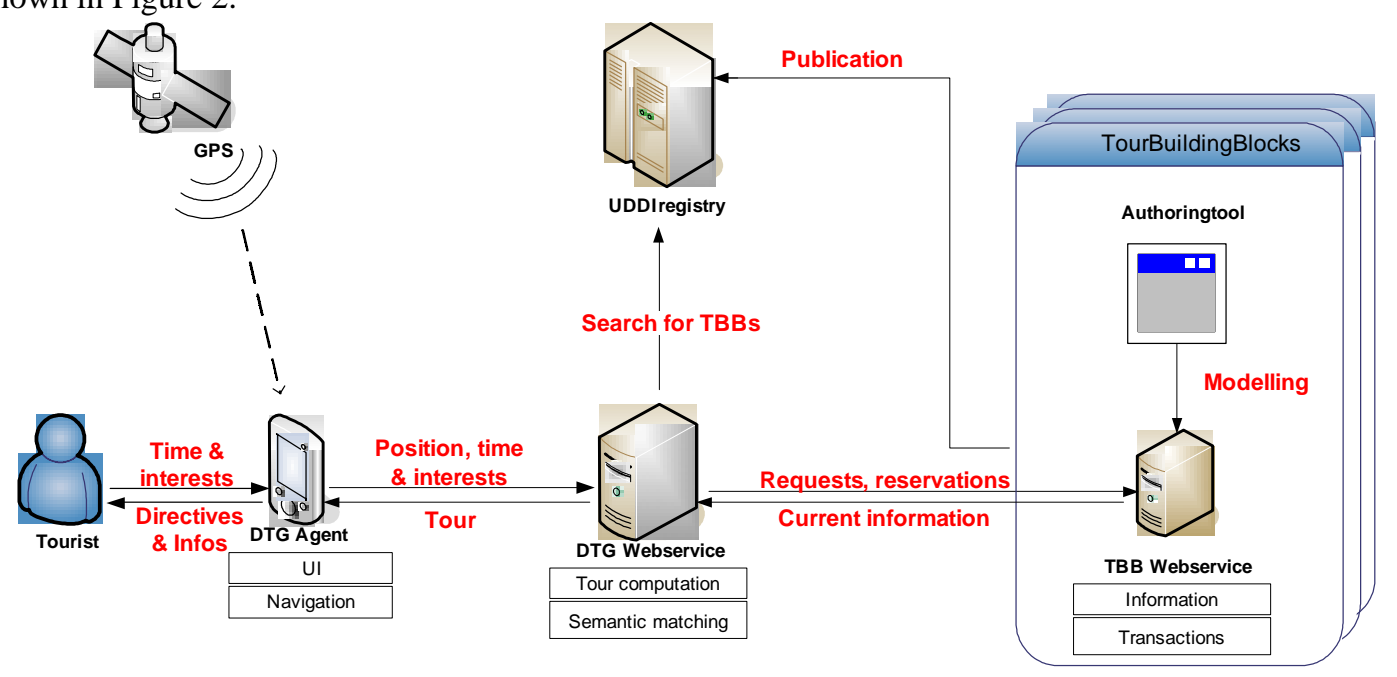

Figure 1. System architecture

At the beginning an ontology will be defined for a single destination. This ontology will have to be extended slightly in order to be used for other destinations in the same region. As the system is being applied to other regions it is important to maintain a hierarchical ontological system in order to enable reuse of the interest profiles. Otherwise a tourist would have to describe his interests from scratch whenever he/she enters a destination in a new region, which at best will lead to very shallow interest profiles.

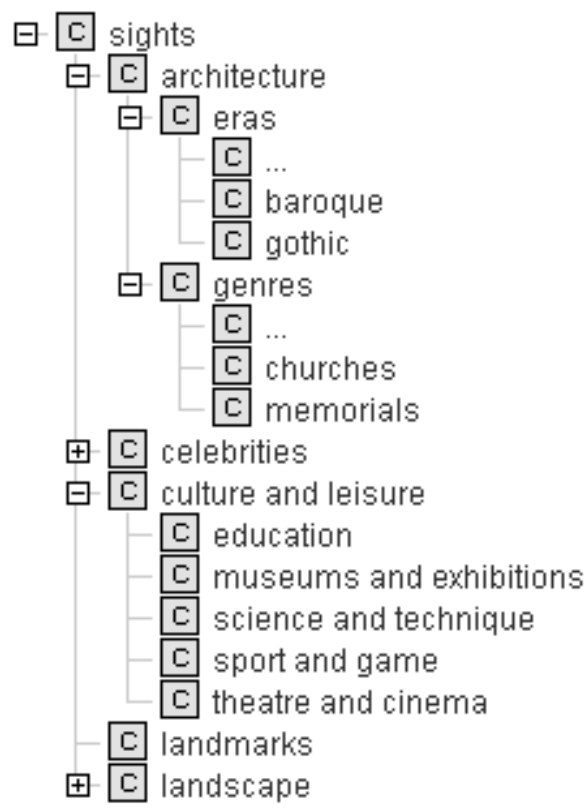

Figure 2. Hierarchical subset

Each category is subdivided. This allows a more precise modelling of interests. For example if a tourist is interested in buildings, he/she can either select a certain type of building like churches, castles and so on, or he/she can opt architectural styles like baroque, art nouveau or others. The idea is that selecting a certain category, the tourist's preference will probably comply with neighbour classes (subclass or parent class) in the ontology as well. The tourist might be interested in related sights too. That means that close-by classes are expected to be semantically similar so that relationships become visible easily.

The TBBs are sorted into this hierarchy by the content providers using an AuthoringTool thereby creating the TBB models. Most TBBs will be listed in multiple branches of the hierarchy, e.g. a church might be listed under architecture/genres/churches and architecture/eras/gothic. The sorting process results in the creation of an XML-profile that contains all chosen categories with all relevant superclasses.

\section{1) Semantic matching}

Based on the ontology, the interest profile and the TBB models are compared by the semantic matching algorithm to compute the degree of similarity. This is expressed in a certain amount of points (IMPs = interest matching points) with the basic value 1 . Therefore the semantic matching algorithm evaluates the hierarchical part of the ontology, which is a directed graph, with a given interest profile. The node presenting this interest is evaluated with 1 . There are two functions the rest of the nodes can be evaluated with, whereas each node is restricted to have exactly one parentnode. Going up, the IMPs of the nodes are divided by two: 


$$
y \leftarrow f^{u}(x)=\frac{1}{2} x
$$

Going down, the sub nodes receive the same IMPs as their parent node:

$$
y \leftarrow f^{d}(x)=x
$$

Presumed node B was chosen as the starting point, an evaluated abstract graph looks like shown in Figure 3 (nodes A - G can stand for a subset of the hierarchy from Figure 2).

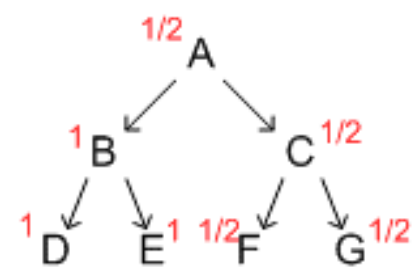

Figure 3. Evaluated graph

For $\mathrm{D}$ and $\mathrm{E}$ function $\mathrm{f}^{\mathrm{d}}(\mathrm{x})$ fits, so these nodes are also valued with 1 . Node $A$ receives $1 / 2$ because of function $\mathrm{f}^{\mathrm{u}}(\mathrm{x})$. $\mathrm{C}$ as a subnode of $\mathrm{A}$ and $\mathrm{F}$ and $\mathrm{G}$ as subnodes of $\mathrm{C}$ are all rated with $1 / 2$ by $f^{d}(x)$.

In practice the interest taxonomy is evaluated like this using the interest profile of the tourist. For each TBB the values of all assigned categories are summed up and result the IMPs for that particular TBB. More details can be found in [25].

2) Tour calculation

After the semantic match algorithm has assigned IMPs to each TBB a tour can be computed. A valid tour is a sequence of TBBs that can be visited within the time allocated by the tourist [12]. The challenge is to compute a valid tour that maximises the IMP in no more than 5 seconds as tourists in mobile context won't be willing to wait much longer. Each TBB has an average duration of visit. Since 20 TBBs with the same start and end point lead to $(20-1) ! / 2=6 * 10^{16}$ possible tours, valid tours can't be cached in advance and thus need to be computed online. Finding the optimal solution for that complex task may take hours of computation depending on the amount of TBBs. That's why an approximation algorithm based on a depth first search that finds a nearly optimal solution within a short time is used.

The depth first search problem means that each TBB which can be part of the tour (or not) has to be combined with the other TBBs whereas the sum of the IMP has to be compared with the maximum. This formally means traversing a tree of possible solutions with backtracking in case remaining time is to short for visiting more TBBs. Supposed that $N$ is the depth of the tree the complexity of such an algorithm would be $\mathrm{O}\left(2^{\mathrm{N}}\right)$ since each combination has to be analyzed to find the one with the most IMP.

Whenever the tourist requests his/her mobile device to compute a tour she/he is most likely standing somewhere within the destination holding the mobile device in his/her hands. Given that situation the tourist won't care too much if the tour presented to him after e.g. 5 seconds has less IMPs than the optimal tour. For most tourists the optimal tour is irrelevant - actually any tour, if the computation takes more than 5 seconds. The challenge is to find heuristics that direct the algorithm to find tours with many IMPs quickly. Therefore the underlined elements of the algorithm were optimized to quickly focus on good approximations of the optimal tour:

\section{Selection of the TBB candidates}

$\rightarrow$ Build up a candidate list by checking whether they can be reached within the remaining time.

\section{Insertion of the new TBB}

$\rightarrow$ Build up a partial tour by inserting TBBs at positions of minimal costs recursively and removing them from the candidate list.

\section{Sorting the candidate list}

$\rightarrow$ Sort the candidate list by the quotient of IMP gain and time (timeInTBB = time spent for staying at the TBB, addTravel $=$ time spent for walking there) loss:

$$
\frac{\text { gain }}{\cos t}=\frac{I M P}{\text { timeInTBB }+ \text { addTravel }}
$$

This leads to more compact tours with shorter distances between the single TBBs.

\section{Pruning of the solution space}

$\rightarrow$ Prune the candidate list to avoid the computation of identical tours.

Different varieties of these heuristics have been combined and compared in several tests. The fine-tuning of these heuristics is a trade-off between capability and runtime. A more capable heuristic tends to consumes more runtime and thus reduces the search space that can be inspected during the available 5 seconds. A more detailed discussion can be found in [25].

3) Restaurant integration

In [23] Schmidt-Belz has shown that restaurants are one of the most important information needs for a tourist. That's why a mobile information system like the DTG which intends for an optimal allocation of local services has to provide the option to integrate a restaurant into the tour plan. This necessity comes up with some problems concerning the calculation algorithm of the DTG.

Generally a restaurant possesses the same properties like an ordinary TBB because it has an address (position), some profile information, an average visit time and so on. But there are additional ones like restaurant-choice constraints (nationality of the kitchen, price class, available beer garden, vegetarian food etc.) and a reservation time span that have to be considered as well. To include the restaurant feature into the DTG tour calculation some general sequences have to be changed.

After the DTG has found all TBBs existing in the destination a second search for restaurants fitting to the constraints given by the tourist must be started. The result list has to be 
sorted according to the degree of compliance between the restaurant profile and the constraints. To enable the DTG algorithm to handle a restaurant in the same way like a TBB it has to receive interest matching points. A very simple approach is to assign the IMPs of the already matched TBBs onto the restaurants whereas the best restaurant result (first place in the list) gets the same amount of IMPs as the best ranked TBB. As it was shown in 2) the IMPs have an important impact on the results of the tour calculation. That's why the user has to weight the importance of the restaurant. In case the restaurant is very important it is planned first which might lead to losses of the most interesting TBBs if they are situated in a distant part of the city. In the reverse case of a low importance of the restaurant, it doesn't have a big influence on the tour calculation because the restaurant is integrated along the path after the tour was calculated. Thereby it is very likely that this restaurant isn't the optimal one for the given constraints.

Another problem is to integrate the restaurant for a certain time which the user had specified. This time constraint could affect the tour calculation significantly but it is important as a reservation of a restaurant table is absolutely time depended. Especially in the peak season such a feature is necessary because a lot of localities don't have any free tables left within the rush hour. The reservation time could easily be considered as an opening time of a TBB (like museum, shop etc.) because a valid tour is a combination of $\mathrm{TBB}$ whereas each is reachable within its opening times.

\section{Tour execution}

Figure 4 displays the complex interactions during the tour execution which is driven by the different contexts and the navigator. They all result in appropriate information like multimedia content, audible user guidance and maps. The context driven interpretation will be described in the first part of this section followed by navigation and tour adaptation which will be covered in the second and third part.

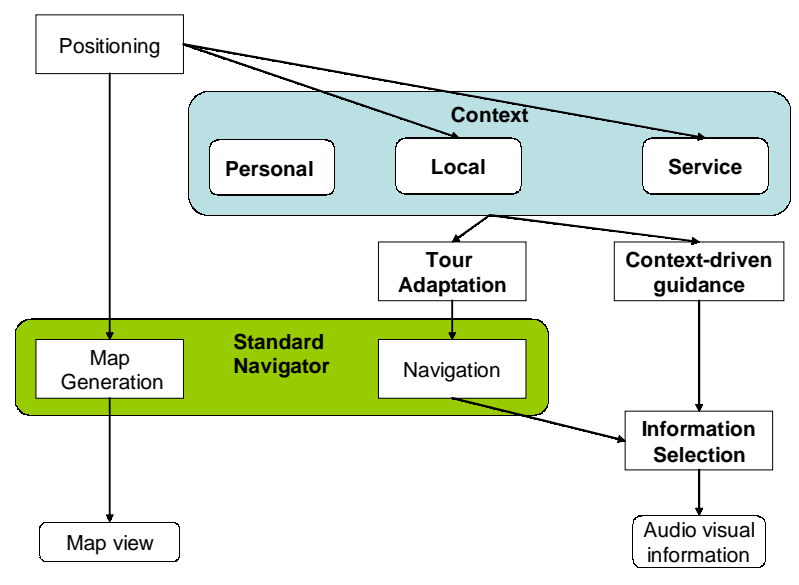

Figure 4. Context-driven tour execution and interpretation

\section{1) Context-driven interpretation}

Based on the tourist's personal context a tour starting at the current position, including sights according to the interests and ending in the given time frame is generated.

Along the way a pre-loader will be called at regular intervals, e.g. 5 minutes, to download all contextual information for the area the tourist can reach within a certain amount of time, like the next 10 minutes. As a side effect this forward looking caching makes the DTG more robust in situation with spotty mobile coverage.

The data is ready for being presented on the mobile device when the tourist reaches the attraction. The presentation continues as long as the tourist keeps standing in front of it. As he leaves the presentation stops and the navigation to the next attraction begins.

As mentioned above, the contexts are first used for the tour computation. The tourist's personal context, especially considering his actual position, interests and time frame, affects the selection of the sights. The contexts are secondly applied for supervising the tour execution. The DTG is always aware of context changes which appear most clearly when approaching a TBB. The following figure shall demonstrate the interactions resulting from that scenario:

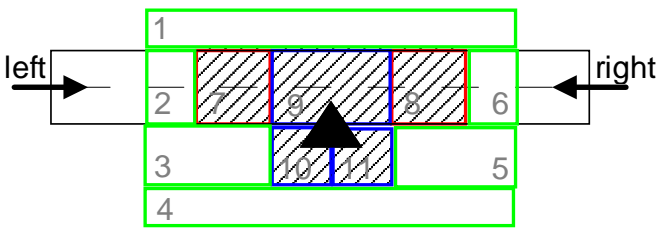

Figure 5. TBB model

The actual attraction is located near a certain street. All necessary data are modelled in a database scheme. This also includes a separation of the area around the TBB into virtual geometric forms. As circles cause overlaps the most suitable ones are rectangles. Furthermore rectangles can be evaluated computationally efficient. For every rectangle the upper left and the lower right coordinates are known. The coordinates 
of the tourist's current position are transmitted by a DGPS receiver. To determine if the tourist is situated in one of the rectangles it needs to be checked whether his/her coordinates are smaller than the upper left and bigger than the lower right ones:

$P^{u p_{-} l e f t}(x, y) \leq P^{\text {tourist }}(x, y) \leq P^{\text {low_right }}(x, y)$

This kind of positioning strongly depends on the accuracy of the delivered GPS-signals which needs to be close to few metres. Else the rectangles may need to be modelled bigger and/or merged together to fewer ones. Entering a rectangle, the following table shows the events which are triggered conditional on the rectangle the tourist has been before:

\begin{tabular}{|l|l|l|}
\hline Entering & from & Action \\
\hline 7 & 2 & $\begin{array}{l}\text { Alert about the forth-coming } \\
\text { TBB, e.g. "On your right you } \\
\text { see ..." }\end{array}$ \\
\hline 8 & 6 & $\begin{array}{l}\text { Alert about the forth-coming } \\
\text { TBB, e.g. "On your left you } \\
\text { see ..." }\end{array}$ \\
\hline 7,8 & 9 & $\begin{array}{l}\text { Alert about the departure from } \\
\text { the current TBB }\end{array}$ \\
\hline $9,10,11$ & - & $\begin{array}{l}\text { Information about the front } \\
\text { side or back side of the TBB }\end{array}$ \\
\hline 2 & 7 & $\begin{array}{l}\text { Navigation towards the next } \\
\text { TBB }\end{array}$ \\
\hline 6 & 8 & $\begin{array}{l}\text { Navigation towards the next } \\
\text { TBB }\end{array}$ \\
\hline 2,6 & - & $\begin{array}{l}\text { Navigation towards the current } \\
\text { TBB }\end{array}$ \\
\hline $1,3,4,5$ & any & $\begin{array}{l}\text { Alert of potential deviation } \\
\text { and navigation back to the } \\
\text { TBB or the route }\end{array}$ \\
\hline
\end{tabular}

Table 1. Actions when approaching or leaving a TBB

The method of context-driven interpretation can be extended by additional user guidance within a TBB itself. Having arrived at a church, the subtour might start at the front door, showing e.g. a picture of its digital reconstruction on the screen of the mobile device. The screen will further present a map showing the single stations around the church with different information sources. The audio hints are using cross references, e.g. "Please enter the church to receive information about the organ." Inside the church, the tourist might hear an mp3-file of a concert. When moving out again the tourist is informed that he will receive historical information about the tower at the backside. With audible instructions supported by arrows on the screen the tourist is guided to the next station. Missing exactness of GPS-signals will have to be balanced by the users' involvement, e.g. the user has to select a photo to indicate her/his current position.

\section{2) Navigation}

The DTG applies a standard navigator installed on the mobile device to navigate from one sight to the next. This navigator is a separate program using offline available geographical data for navigation and a stored map data for showing instructions and routes on a map. Furthermore the navigator gives instructions via audio to avoid the user holding the PDA in field of view all the time.

\section{3) Tour adaptation}

The DTG relies on GPS data to determine the tourists' position. Especially in urban regions with high buildings (that cause shading and reflections) these data can lead to inexactnesses up to several meters [7] from the actual position. Hence the navigational instructions won't be exact which may disorientate tourists within a town centre with many close-by alleys. Furthermore town centers provide many distractions like shops, cafés and more that will catch the tourists' attention. Changing user behavior due to these external influences will affect the execution of the tour-plan. These influences can't be considered in advance and need to be reacted on in real-time to satisfy the user or she/he will soon switch off the tour guide. Hence the plan mustn't be static but dynamic so that an adaptation of the tour can be done whenever required.

The difficulty herein is to adapt the plan according to the tourists changing behavior and ambitions with minimal interaction mainly by watching the tourists' actions and guessing her/his intentions. Here some kind of intelligence is needed in order to decide between two cases. If the tourist is unable to find the right way then she/he needs helping navigational instructions but if the tourist knows the right way and wants to go somewhere else instead then the application needs to stop any navigation instructions until the new aim becomes clear so that additional support can be provided if possible. Hence the tour adaptation process is indispensible to provide a truly personalized experience.

There will be adaptation on three levels:

1. The individual tour needs to be adapted to the actual walking speed and progress. The timeframe of the tourist is the given constraint that mustn't be exceeded to get her/him to the desired endpoint in time. This adaptation works as a revaluation that computes a new tour from the current position with the remaining amount of time whenever necessary.

2. The models of the TBB need to be adapted too in order to reflect the average time the tourists spent in the vicinity of this TBB.

3. The personal interest profile might need to be updated, in case a tourist stays shorter than the average tourist at certain TBB, which might indicate a smaller level of interest than originally assumed and on the contrary if the tourist spontaneously visits a certain TBB, which was assumed to be of no interest.

The main challenge is to evaluate the state of the tourist to 
apply a certain rule and to adapt the tour to the new conditions. State transitions then cause certain adaptation activity. Potentially any change of the user state or the context can be a reason to have to adapt the tour. User state (current position and movement) and environmental properties (near attractions) can be determined at any time. Combining all information like current user state, past user states and the actual context, the tourists' intentions can be estimated too. Then a decision about the necessity to adapt the tour considering the needs of the tourist is possible. The following steps are necessary:

1. A state model for a tourist including her/his intentions and actions has to be constructed: The tourist can have the following states concerning the progress of the tour and her/his movement (the expected state, which doesn't require any adaptation of the original tour plan is underlined):

\section{- $\quad$ On time / late / fast \\ - Walking / Strayed / Resting}

2. The actual states can then be determined to estimate the intention based on the observations, e.g. movements and locations. A map might be used to map a position to a location of a certain activity. If there is a high uncertainty in the estimation, the tourist has to be involved by providing her/him options to select her/his intention.

Information most appropriate to the determined intention can be delivered to the tourist afterwards.

\section{Field trial}

The article so far described the concept of the DTG. Before its final development basic usability issues and the practical relevance should be examined by the following experiment.

The field study, conducted in Görlitz in June/July 2005, was designed to answer the following questions in particular: (1) The DTG computes an optimal tour according to the interest profile of a tourist. Therefore the most fundamental question is: Is it possible to build a mobile system that collects information from the tourist, which is sufficient to select attractions? (2) In case an effective mobile "interest gathering" capability can be built, the next question is: How diverse are the interests of tourists? Are the interest profiles so similar anyhow that the optimal tours hardly differ and thus the tourists are best served by following the beaten tracks? In case only 2-3 prototypical interest profiles exist, then the corresponding number of standard tours would be sufficient. (3) The last and most important question for any form of pervasive computing or ambient intelligence is: Does the additional contextual information affect the spatial behavior of tourists or does the DTG merely increase the ambient noise?

\section{A. Experimental setup}

Before the experiment could start, about 80 sights of the city
Görlitz were modeled semantically, which means that they had to be assigned to classes of the ontology - the classification of all tourist attractions. Additional pictures and describing text were collected for each sight in order to provide fair means for the task of ranking concrete sights.

For a timeframe of about 4 weeks, 234 tourists were given an MDA, which is a PDA type mobile device with an integrated mobile phone. Their first task was to complete a questionnaire asking for age, gender and previous experience with certain computing and communication technologies. The second task was to use one of three applications to express their interests:

\section{- Hierarchical browser:}

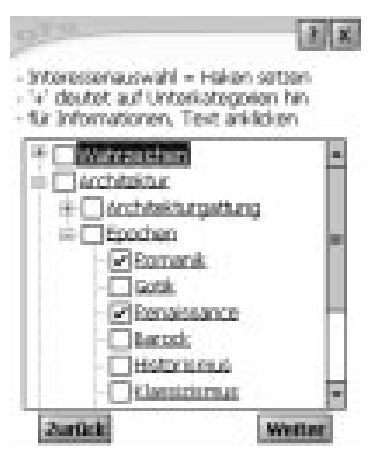

The hierarchical structure of the ontology is visualized by a tree view element. The user can select any category she/he is interested in by checking the boxes. The advantage is that everything can be displayed on a single screen which turns into a disadvantage at the same time as small fonts have to be used and scrolling becomes necessary when expanding the tree.

- Inspirational images:

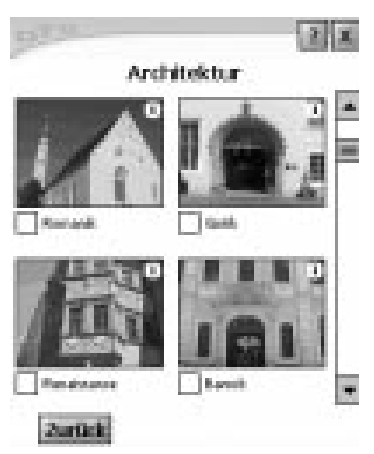

The hierarchy is presented by iconic images for each level. These images shall inspire associations causing positive or negative feelings with each term. The pictures can be maximized and information for each term is offered too. The advantage here is the visualization by pictures and symbols. However these images make a lot of screens necessary and therewith lead to a difficult orientation between the levels.

\section{- Main categories}

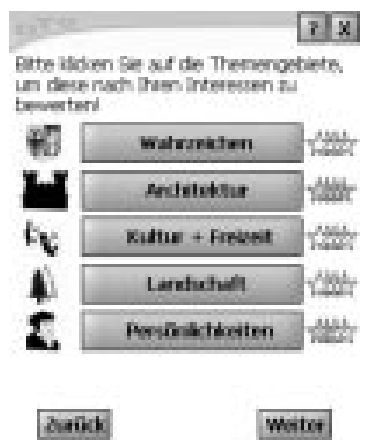

Only the main categories are provided for selection. Selecting one category will open a pop-up window to give a percentage value to express the intensity of the interest displayed by a certain amount of colored stars.

Table 2. Screenshots of interest specification GUIs 
After the participants had expressed their interests using one of the three methods the semantic matching algorithm rated all available sights appropriately. The two best rated, the two worst rated and two medium rated sights were picked out and displayed on the PC in random order. The tourist was then asked to rank the six concrete attractions using descriptions and pictures provided for each. The purpose was to find out the method that was able to predict the behavior of the tourist in ranking the concrete sights. In other words it was searched for the preference elicitation method, which produced the highest correlation between predicted ranking and the ranking created by the tourist. If a tourist brings the sights into the same ranking as the algorithm, then the highest possible correlation has been achieved.

\section{B. Results}

The age of the 234 participants ranged from 13 to 78 , while the average age was 47 years. However, the modal age was 60 and 63 what gives a better impression on the actual age pattern. $60 \%$ were male. More than $2 / 3$ of the participants stated a regular use of the PC. Still more than $1 / 3$ often works with the internet and a handy, while almost nobody uses an MDA. $90 \%$ own a PC or a handy.

\section{1) Interaction durations}

Table 3 shows the duration of the interest elicitation process in mobile context, the number of clicks and the duration a tourist interacted with a single panel. Surprisingly for all three methods the results are similar. Tourists spent about 2 minutes or less specifying their interests with about 9 seconds per screen using a total of 22 clicks.

\begin{tabular}{|l|l|c|c|c|}
\hline \multicolumn{2}{|l|}{ Method } & Tree & Images & Categories \\
\hline $\begin{array}{l}\text { Duration of } \\
\text { elicitation } \\
{[\text { [min] }}\end{array}$ & Mean & 2.03 & 2.12 & 2.03 \\
\cline { 2 - 5 } & Median & 1.44 & 1.28 & 1.50 \\
\hline Clicks & Mean & 16 & 29 & 21 \\
\cline { 2 - 5 } & Median & 12 & 13.5 & 19 \\
\hline \multirow{2}{*}{$\begin{array}{l}\text { Duration } \\
\text { of panel } \\
\text { view [sec] }\end{array}$} & Median & 5 & 9 & 8 \\
\cline { 2 - 5 } & Min & 2 & 3 & 4 \\
\cline { 2 - 5 } & Max & 10 & 20 & 13 \\
\hline
\end{tabular}

Table 3. Preference elicitation: Duration and clicks

The time spent for viewing a single screen differs up to four times, since the navigation leads the user to the same screen several times. The screens for the image version are the most complex ones providing pictures, text and scrolling opportunities. Thus they have been viewed longest. But as the median values and tendencies for all three methods hardly differ they potentially define the attention span of a tourist of this age in mobile context.
The following diagram displays the distribution of clicks used by the tourists to specify their interests. The tree and category method have one well defined peak, whereas the image method shows 2 of them. Many tourists remained in the top-level, indicated by the first maximum, and therefore needed very few clicks. But those who also used the deeper levels needed the most clicks of all shown by the second maximum.

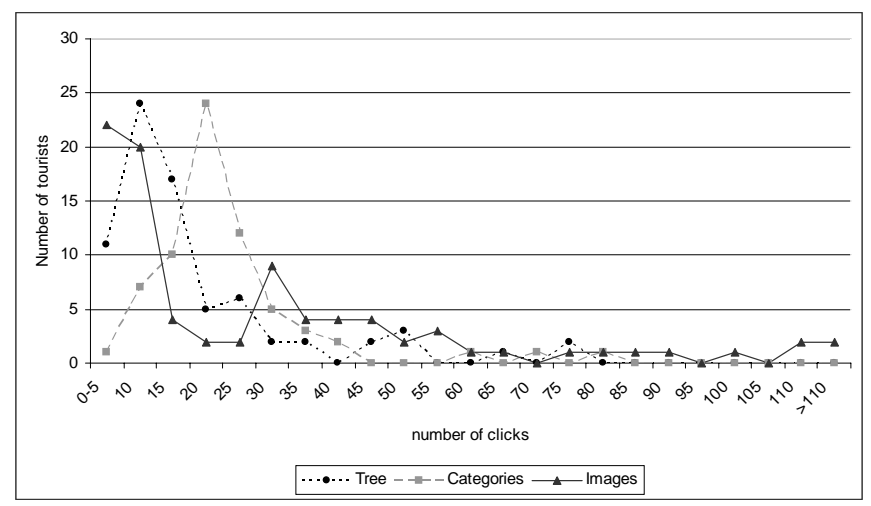

Figure 6. Number of clicks per method

Not surprisingly the number of clicks and the duration of the interest gathering process are closely correlated with 0.82 .

\section{2) Interest selection}

Evaluating the gathered interest profiles allowed an insight into the selection behaviour. In every method the category "architecture" or its subcategories have been chosen most. That was expected since Görlitz offers many architectural sights that most tourists are coming for. Landmarks have been selected very often too, which is a good choice for tourists not knowing anything about the destination.

The most important question here is how detailed the interests of tourists are and how detailed they are willing to specify them. Five main categories were offered. Close to $40 \%$ of the tourists selected interest terms out of at least 2 different categories and about 4 different interests in general. The diagram in Figure 7 displays the deepest levels the tourists reached when specifying their interests for the tree and image version.

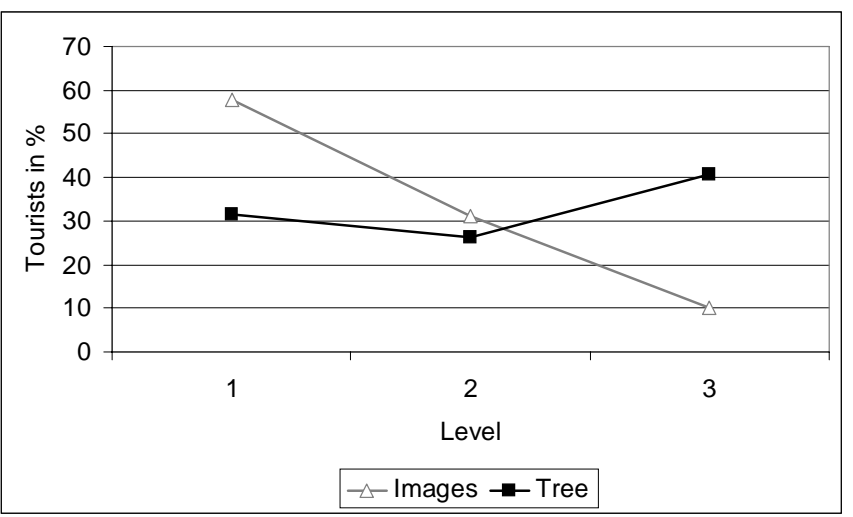

Figure 7. Level-distribution of the interest selections 
For both these methods (image and tree) a selection of interests of deeper levels was possible. And indeed further analysis indicated that about $70 \%$ of the tree explorer users and $40 \%$ of the image version users made use of this and specified more detailed interests than only top-level categories.

But this analysis also shows that more than half of all tourists using the inspirational image method didn't specify interests in any deeper level than the first. The usage pattern was thus very similar to the much simpler method using only main categories as they mainly selected top-level categories. In contrast the third level was used most often from tourists working with the tree explorer.

The evaluation of the screen shots indicates that most tourists didn't realize that clicking on a category name or its icon reveals subcategories displayed by concrete images, though it was mentioned on the screen. The problem of the inspirational method on a mobile device is that in order to provide choice at least two images need to be shown. To minimize scrolling four images were picked. Four images don't leave space to include visual clues to the hierarchical nature of the selection. In order to clarify whether more tourists would make use of the possibility to express their preferences in more detail all three methods will be ported to the web where the images and visual clues can be presented more effectively.

\section{3) Correlation}

The interests selected by the tourist served to rank the available attractions by the algorithm. 6 concrete sights of that list had to be ranked explicitly by the tourist. The similarity between user defined rankings and algorithmbased rankings can be expressed in the form of rank order correlations. The best result is an identical ranking (value 1), the worst one is an opposite list (value -1). A value of 0 signifies that there is no recognizable correlation. The correlation value is determined by the formula of Spearman which compares two ranked lists according to Lowry [19].

$$
r_{s}=1-\frac{6 \times \sum_{i=1}^{n} d_{i}^{2}}{n \times\left(n^{2}-1\right)},
$$

with $\mathrm{n}=$ number of elements, $\mathrm{d}=$ difference of the element positions, $\mathrm{i}=$ index

The difference of the positions of each sight in both lists is calculated and squared. The condition is that the elements must be ordered ordinal. That means that there is a significant difference between the first and second element and so on. In this case there might be sights at adjacent positions having received the same amount of points. Then the difference is set to 0 , where else it is 1 .

The correlation results are listed in Table 4. From a correlation perspective the median correlation for the relatively simple method using five main categories and the imaginative method using images are equally effective in capturing the interests of the tourists. The last method is using a Windows Explorer style hierarchy browser which additionally holds the highest standard deviation. The median correlation values also show that more than half of all tourists have reached a correlation higher than 0.5 for all methods (even higher than 0.6 for the image and main category version).

\begin{tabular}{|c|c|c|c|c|}
\hline \multicolumn{2}{|l|}{ Method } & Tree & Images & $\begin{array}{l}\text { Cate- } \\
\text { gories }\end{array}$ \\
\hline \multirow{3}{*}{$\begin{array}{c}\mathrm{r}_{\mathrm{s}} \\
\text { coefficient }\end{array}$} & Mean & 0.47 & 0.48 & 0.52 \\
\cline { 2 - 5 } & Median & 0.54 & 0.6 & 0.6 \\
\cline { 2 - 5 } & $\begin{array}{c}\text { Std de- } \\
\text { viation }\end{array}$ & 0.44 & 0.39 & 0.36 \\
\hline
\end{tabular}

Table 4. Preference elicitation: Correlation

Considering the maxima of the correlation distributions in Figure 8 reveals a value of 0.8 or higher for all three methods what means that for the majority of the tourists the recommendations are pretty good. Only very few reached a negative correlation.

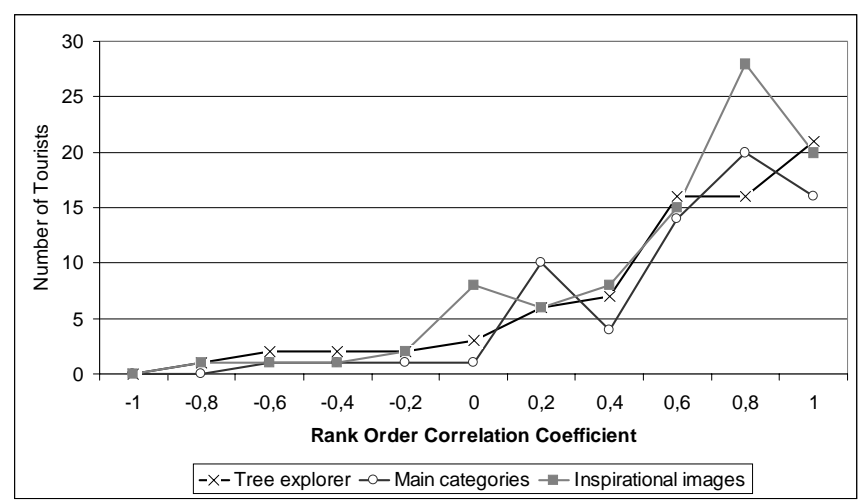

Figure 8. Distribution of correlations

Surprisingly the amount of clicks as well as the amount of time doesn't have a positive effect on the spearman coefficient. The correlation for both dependencies is -0.1 , which means that there is little dependency. One might now reason that 2 minutes must be enough to elicit preferences from a tourist. At least with the three methods used in this field study any further effort doesn't lead to an improvement of the ability to predict the selection of attractions.

\section{4) Entropy calculation}

Given the values of the rank order correlation coefficient it can now be assumed that the semantic matching algorithm is able to rank the attractions based on the gathered interest profiles according to the desires of the tourist. Nonetheless an ambient intelligence device computing individual tours might not be necessary, since the interests of the tourists are pretty much the same or fall into a couple of well-defined prototypical interest profiles. Therefore the next crucial question is how diverse are the gathered interest profiles? 
A way to assess the diversity independently of the actual distribution is to measure the entropy. Therefore each profile is interpreted as a combination of interests. Each combination has a certain probability of occurrence, which can be determined by dividing the frequency of each profile by the number of profiles in total. The single probabilities are then used to compute the entropy:

$H=-\sum_{k=0}^{L} p_{k} * \log _{2} p_{k}$,

with $\mathrm{L}=$ number of profiles, $\mathrm{p}=$ probability of profile $\mathrm{k}$

The computed entropies are displayed in Table 5. The entropy values measure the average information content per profile in bits. If all profiles were identical the entropy would be zero meaning that they don't contain any new information. If all profiles are different the entropy is the dual logarithm of the number of profiles. As the values are between $85 \%$ and $98 \%$ of the maximal entropy most profiles are unique and thus do contain new information than other profiles already provided.

The entropy calculation for the interest profiles as a whole showed that the overwhelming number of profiles is different. However it might still be the case that there is considerable overlap between the interest profiles. Therefore each profile was compared against the other profiles, determining how many elements are identical. The average amount of identical elements is expressed as a percentage listed in Table 5.

\begin{tabular}{|l|l|l|l|l|}
\hline \multirow{2}{*}{ Method } & \multicolumn{3}{|c|}{ Entropy } & \multicolumn{1}{c|}{ Overlap } \\
\cline { 2 - 5 } & Max & Actual & Relative & Min-Max \\
\hline Tree & 6.23 & 5.76 & 0.92 & $3 \%-53 \%$ \\
\hline Images & 6.49 & 5.56 & 0.85 & $1 \%-40 \%$ \\
\hline Categories & 6.09 & 5.97 & 0.98 & $17 \%-34 \%$ \\
\hline
\end{tabular}

Table 5. Diversity of interest profiles

The right side of Table 5 lists the overlap number. For the tree version up to $53 \%$ of overlap is given meaning that in the average between $3 \%$ and $53 \%$ of the classes in an interest profile are common with another interest profile. Despite this partial overlap in interest elements between each other, the degree of difference between the interest profiles still makes it difficult to split them into similar groups around a prototypical interest profile as discussed in the next part 'Clustering'.

Furthermore the entropy of the distribution of selected interests within the ontological hierarchy can be determined. It gives an impression if the tourists select the same nodes within the same branch or if the selections are spread evenly across the whole tree structure. The absolute numbers of selections of each interest term were taken to calculate the entropy for each level of the hierarchy. What can be seen is that the interests are indeed very individual and tourists do not select the same things at all as the entropies reach more than $90 \%$ of a best case evenly distribution.

\begin{tabular}{|l|c|c|c|}
\hline Image version & $\begin{array}{l}\text { Actual } \\
\text { Entropy }\end{array}$ & $\begin{array}{c}\text { Max } \\
\text { Entropy }\end{array}$ & $\begin{array}{l}\text { Relative } \\
\text { Entropy }\end{array}$ \\
\hline Level 1 & 2.28 & 2.32 & 0.98 \\
\hline Level 2 & 3.7 & 4.0 & 0.93 \\
\hline Level 3 & 3.98 & 4.17 & 0.95 \\
\hline
\end{tabular}

Table 6. Diversity of the interest selection at various levels of the ontology using the image version

\section{5) Clustering}

Having proven a high diversity of the interest profiles, another approach is to find out possible similarities between them and to try to constitute groups of tourists with similar interests, so called clusters. Clusters have a high intra-class similarity but a low inter-class similarity [15]. A basic value to express the degree of similarity between two elements is their distance. The aim is to determine the distances between the profiles to be able to make a statement about their similarity. The distance between two profiles depends on the distance of their elements which makes some definitions necessary:

1. Dist1 $(e 1, e 2) \rightarrow$ The distance between 2 interest elements (of one profile)

It returns the number of branches between both interest elements within the ontology.

2. Dist2 $(e, p) \rightarrow$ The distance between an interest element and a profile

It returns the minimal value of distances between the single interest element and each interest element in the profile

$$
\operatorname{Dist} 2(e, p)=\operatorname{MIN}\left(\forall_{i е \ni p}:(\operatorname{Dist1}(e, i e))\right)
$$

3. $\operatorname{Dist3}(p 1, p 2) \rightarrow$ The distance between two profiles It returns the maximal value of comparing profile 1 with profile 2 and profile 2 with profile 1

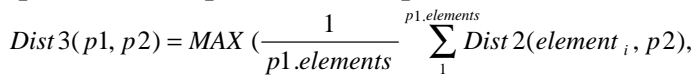

$$
\begin{aligned}
& \left.\frac{1}{p 2 . e l e m e n t s} \sum_{1}^{p 2 . e l e m e n t s} \text { Dist }^{2}\left(\text { element }_{i}, p 1\right)\right)
\end{aligned}
$$

The distances for each profile to any other profiles are determined which results in a matrix.

Based on these distances the clustering was done by the following algorithm:

Foreach profile $\mathrm{pl}$

Determine profile 22 with the lowest distance towards p1

If profile 22 belongs to a group

$$
\text { Else }
$$$$
\text { Add profile pl to that group }
$$

Create a new group with $p 1$ and $p 2$

This returned the following amount of groups: 


\begin{tabular}{|l|l|l|}
\hline Method & \# of groups & $\begin{array}{l}\text { average \# of profiles } \\
\text { in group }\end{array}$ \\
\hline Tree & 30 & 2.5 \\
\hline Images & 40 & 2.3 \\
\hline Categories & 31 & 2.2 \\
\hline \multicolumn{3}{|c|}{ Table 7. Grouping results }
\end{tabular}

The algorithm creates pairs or groups of profiles, putting the closest related profiles together. The average number of profiles in such a cluster is small. The clusters are mostly pairs what means there are no proper clusters to be found.

For obtaining fewer and bigger groups the profiles would need to be more categorical. But a number of 30 groups and higher with less than three typical profiles in it can't be considered as clusters, because it's not feasible to prepare standard tours for 30 clusters in advance. As there are very few profiles being closely related to each other an individual interest elicitation is compulsory.

\section{Spatial behaviour analysis}

The spatial behavior of tourists is of tremendous economic importance for a tourist destination. Stakeholders such as city councils have a strong interest in evaluating spatial behavior to avoid misallocation of their scarce resources. This analysis was done to prove that interest based tours have a strong effect on the allocation of tourists compared to the allocation when using the beaten paths.

\section{A. Related approaches}

Traditional ways of measuring tourists' spatial behaviour rely on diary data completed during a visit or on questionnaires completed after the actual tour or trip. The problem with the first method is that it is highly intrusive, whereas the latter relies on the honesty and memory capacity of people when providing their information. It is quite unsure how credible these data are. The ideal would be to $\log$ the movement of a tourist and to measure the time without letting the tourist take note of it. Several such methods have been implemented in the context of transportation and retail studies:

- Dijkstra, Jessurun and Timmermans [6] implemented a model that simulates the movement of pedestrians by agents. Because of defined rules, the agents either move or wait within different cells. They visualise possible interactions of pedestrians in crowded areas.

- Shoval and Isaacson [24] compared geographic information systems like GPS to land-based tracking systems; these are units sending signals to antenna stations that calculate the position, by measuring the movement of pedestrians. The main advantages of GPS are the worldwide ability, little costs and exacter positions, whereas land-based tracking systems have the advantages of being unaffected by the weather and work also well in urban regions and indoors.

- Larson, Bradlow and Fader [18] analysed the paths of shoppers in a supermarket with RFID tags located on their shopping carts. The tracked pathways were clustered to find out typical routes through a grocery store.

\section{B. Current state analysis}

A parallel study used GPS receivers to track the changes of real tourists' positions together with a timestamp. Analyses of the track logs identified the most and longest visited places as well as the tourist distribution in general. Therefore the entire area was divided into equally sized cells. The number of tourists who visited a certain grid cell was displayed by certain colours in a map shown in Figure 9. This is the current state of tourists' distribution.

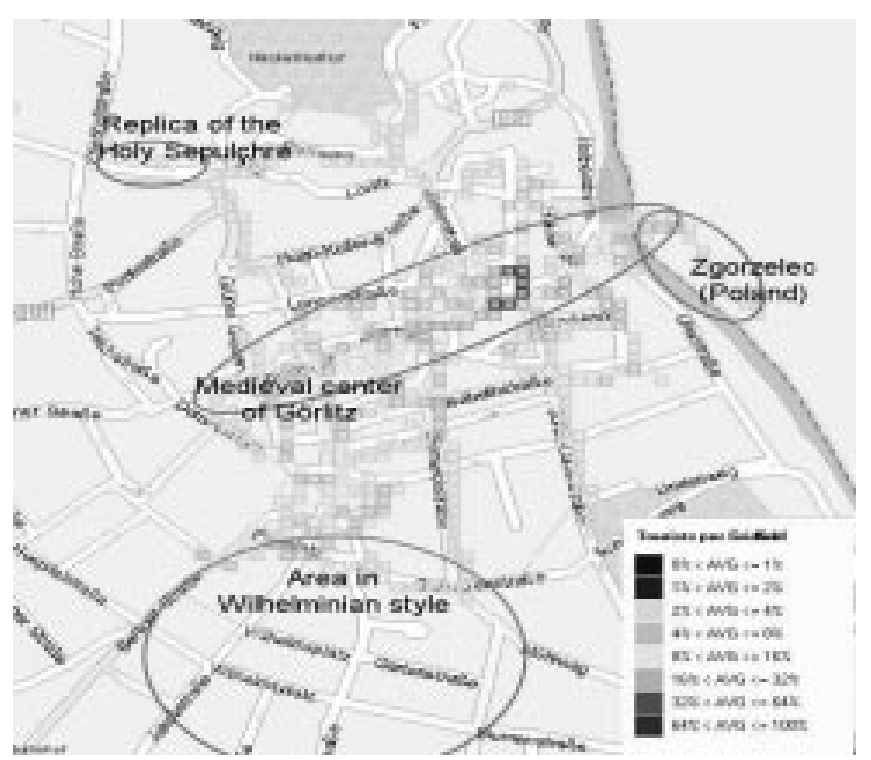

Figure 9. Actual distribution of tourists

Most tourists move within a limited area and very attractive sights apart from that area are visited only by few. Two circles mark two important architectural attractions: One of the most consistent and largest ensembles of buildings in Wilhelminian style in Europe and a replica of the Holy Sepulchre. Both are architecturally important, but nonetheless rarely visited even by architecturally interested visitors. The map indicates a clear need for marketing (e.g. creating awareness) and management (e.g. better signage) to diversify tourist flows at the destination.

The DTG as an information system is built to enable tourists to discover sights or activities that suit their personal tastes. In order to assess the effectiveness of this and other more traditional marketing measures, spatial distribution metrics can be used. A density map provides insight into the spatial distribution of tourists. In order to compute a density map the map is divided into an equal grid (as done to generate the figure above). If a track crosses a cell the number of visitors 
for that cell is incremented. After all tracks have been analysed the number of visitors to a single cell is divided by the total number of visits to all cells in order to compute the relative number of visitors to each cell.

For a certain time period the relative number of visitors $\mathrm{RNoV}$ to grid cell(i,j) can be defined as:

$R N o V(i, j)=\frac{\operatorname{NoV}(i, j)}{T N o V}$

with $\operatorname{NoV}(i, j)=$ the number of individual visitors to cell $(i, j)$ and $\mathrm{TNoV}=$ the total number of visits to all cells being tracked in this time period to have a common basis. Obviously $0 \leq R N o V(i, j) \leq 1$ is valid.

Shannon has developed a formula to calculate the amount of disarrangement in a system - the so called entropy [19]. This formula was adapted for the purpose of spatial measurement by replacing the probabilities through the RNoV values of the several grids. As a result the spatial distribution metric SDM for a given time period, a destination and a set of tracked tourists was defined as follows:

$$
S D M=-\sum_{\forall i, j} R N o V(i, j) \log _{2}(R N o V(i, j))
$$

SDM reaches its maximum if the $\mathrm{RNoV}$ values of all grids have an equal number. That maximum is determined by $\log _{2}(I * J)$ with $I$ as the number of rows and $\mathrm{J}$ the number columns of the grid. A system is situated optimal if all grids have an equal amount of RNoV. In order to indicate how close the SDM approximates the optimum, absolute values need to be divided by the maximal value to receive the Relative Spatial Distribution Metric, which is defined as:

$$
R S D M=\frac{S D M}{\log _{2}(I * J)}
$$

The result set of the RSDM value is defined as follows: $0 \geq R S D M \leq 1$ whereas 0 indicates a very high concentration and 1 certifies that the tourists are spread equally. The following table exemplifies some possible distributions for a grid of four cells and the resulting RSDM values. It clearly becomes obvious that RSDM is a suitable

\begin{tabular}{|c|c|c|c|c|c|}
\hline \multicolumn{2}{|c|}{$\operatorname{NoV}(\mathbf{i}, \mathbf{j})$} & RSD & \multicolumn{2}{|c|}{$\operatorname{NoV}(\mathbf{i}, \mathbf{j})$} & \multirow{3}{*}{$\begin{array}{r}\text { RSDM } \\
0\end{array}$} \\
\hline 10 & 10 & \multirow{2}{*}{1} & 10 & 0 & \\
\hline 10 & 10 & & 0 & 0 & \\
\hline 10 & 1 & \multirow{2}{*}{0.41} & 10 & 5 & \multirow{2}{*}{0.75} \\
\hline 1 & 0 & & 5 & 0 & \\
\hline 10 & 5 & \multirow{2}{*}{0.9} & 10 & 8 & \multirow{2}{*}{0.99} \\
\hline 5 & 2 & & 8 & 7 & \\
\hline
\end{tabular}
metric to measure the equality of the spatial distribution:

Table 8. RSDM calculation example

Table 9 shows the calculated SDM values for Görlitz based on the grid and amount of visitors shown in Figure 9. The
RSDM of 0.45 gives an impression that the actual distribution is far from being optimal. Actually it indicates a high concentration at some points in the city. By means of this value the effect of diverse marketing methods can be evaluated easily if behaviour is tracked before and after measures are set in place.

\begin{tabular}{|l|r|}
\hline \multicolumn{1}{|c|}{ Situation in Görlitz } & \multicolumn{1}{c|}{ SDM } \\
\hline Current & 6.3 \\
\hline Maximum & 14.04 \\
\hline Relative (RSDM) & 0.45 \\
\hline
\end{tabular}

Table 9. Spatial Distribution Metric (SDM)

\section{Simulated behaviour}

Ideally the tour of a tourist would be tracked first without and then with the DTG. However a destination like Görlitz doesn't allow for two independent subsequent tours. Therefore the best possible method is to capture the spatial behaviour of different tourists with and without the DTG. Figure 9 in the previous section shows the spatial distribution of the tourists gathered during a second experiment within the field trial in the summer of 2005. In the summer of 2006 tourists will be able to execute their tours using the DTG.

However during the first field trial 234 interest profiles were collected. In order to assess the impact of the DTG these profiles were used to calculate individual tours. The durations of the tours were distributed equally to the determined durations during the observation of tourist behaviour. This is a conservative approach, since the tours were rather short due to a central point where the tracking gears were handed to the tourists who probably had already completed a part of their tour at that time. The method works as follows:

\section{For every gathered interest profile \\ a. Rank the TBBs available in the TBB DB \\ b. Compute a tour for $[1 / 2,1,2]$ hours

i. $\quad$ Interpolate the tour
ii. $\quad$ Simulate the tour
iii. $\quad$ Collect traces

2. Analyse the traces using the grid

Figure 10 presents the same fragment of the map like shown in Figure 9, assumed the tourists followed the tours based on the 234 profiles and recommended by the DTG. Again the colours of the grids indicate the percentage of tourists reaching each cell. Since no quantitative data about the start and end points of tours is available for Görlitz, they were chosen randomly for the simulations. In the course of the simulation studies it became clear that in order to get tourists to an area the following conditions must be met: (1) the area needs to offer a certain density of attractions, (2) all need to be modelled appropriately and (3) a system like the DTG must make this information available to the tourists. 


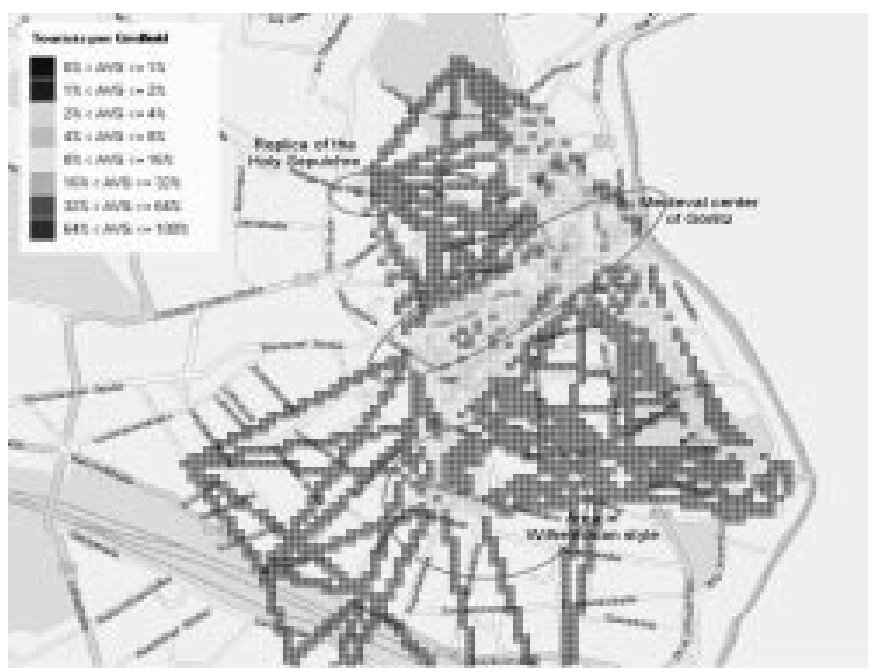

Figure 10. Simulated spatial behaviour

Especially the area near to the waterside street and the city park are better visited. And there are a lot more tourists coming to the area which is built in Wilhelminian style. Even some sights behind the trail station are attended. All together a clear improvement is visible.

The RSDM value for this new distribution shown by Table 10 compared to the current one shown by Table 9 further emphasizes this improvement of the spreading of the tourists. But the RSDM of 0.6 also indicates that the situation is still not an optimum. Though a completely even distribution will never be achieved, adapted marketing concepts may help to lead tourists to more various places and thus further stepwise improve the distribution over a wider area of the city.

\begin{tabular}{|l|r|}
\hline \multicolumn{1}{|c|}{ Situation } & \multicolumn{1}{c|}{ SDM } \\
\hline Simulated & 8.4 \\
\hline Maximum & 14.04 \\
\hline Relative & 0.6 \\
\hline
\end{tabular}

Table 10. Spatial Distribution Metric (SDM)

\section{Future research}

In the summer of 2006 a second field study will be conducted in Görlitz enabling the tourists to use the DTG as an MDA application. The equipment again comprehends a GPS receiver, first to provide navigational guidance and second to the log the tourists' paths. The target is to evaluate the practical relevance and usability of all presented concepts. The same map used to show the present and a simulated distribution can be generated to visualize the distribution of tourists achieved by interest based tours with the DTG. A comparison of both maps will reveal the actual improvement.

So far the focus has been on the interactions of a single person with the DTG. In reality the percentage of tourists traveling alone is small compared to couples, families or groups. As proposed by Franke [9] the concept of the DTG needs to be extended to serve groups of tourists jointly discovering a destination. For this the interest profile of the group has to be synthesized from the individual ones. The decision process of the group to determine the tour needs to be supported, navigation instructions and interpretational information need to be supplied to the group and tour adaptation needs to be enhanced to deal with choices made by individual members. Thus, an improved version of the DTG needs to provide means to support the group management and to provide options to allow for individual freedom as well as group experience.

\section{Conclusion}

The DTG uses context-driven technologies in order to create highly personalized adaptive tours. Independent of location and time it determines the necessary information by detecting and interrogating available web services. It provides complex user guidance by providing navigation instructions and by offering the right information at the right time and place. Permanent tracking of the tour progress considers external influences to adapt the tour.

Semantic technology and an approximate heuristic tour computation algorithm enable tourists to enjoy a destination according to different contexts, which includes their interests, available time, actual position and environmental conditions. Also important is the fact that the DTG will help to spread the tourists more evenly across the destination and give exposure to a much wider set of services. Three different methods to elicit generic preferences were compared in a field trial in Görlitz. Semantic matching based on profiles gathered by the best methods was able to predict the ranking of concrete sights by $50 \%$ of the tourists with a rank order correlation of better than 0.6. An in-depth analysis of the gathered generic interest profiles indicated that they are surprisingly diverse. Finally an experiment having compared the current spatial distribution of tourist in Görlitz with one based on simulated tours using the gathered interests and tour durations indicated that context-aware information will help to enjoy a destination at its full potential.

\section{Acknowledgment}

This project is part of VESUV in cooperation with Siemens AG, Microsoft's European Innovation Center (EMIC) and Fraunhofer Institute IGD. VESUV is supported by the Federal Ministry for Economics and Labour (BMWA).

\section{References}

[1] Abowd, Gregory D.; Atkeson Christopher G.; Hong, Jason; Long, Sue; Kooper, Rob; Pinkerton, Mike (1997): Cyberguide - a mobile context-aware tour guide, Baltzer/ACM Wireless Networks. 
[2] AgentCities (2004): http://www.agentcities.org

[3] Cheverst, Keith; Davies, Nigel; Mitchell, Keith; Friday, Adrian; Efstratiou, Christos (2000): Developing a Context-aware Electronic Tourist Guide: Some Issues and Experiences, Distributed Multimedia Research Group, Lancaster University, UK.

[4] Poslad, Stefan; Laamanen, Heimo; Malaka, Rainer; Nick, Achim; Buckle, Phil; Zipf, Alexander. „CRUMPET: Creation of user-friendly mobile services personalised for tourism", in Proceedings of 3G 2001.

[5] DGPS

http://www.wsrcc.com/wolfgang/gps/dgps-ip.html;

Wolfgang S. Rupprecht.

[6] Djikstra, Jan; Jessurun, Joran; Timmermans, Harry (2001): "A Multi-Agent Cellular Automata Model of Pedestrian Movement" Pedestrian and Evacuation Dynamics, Schreckenberg and Sharma (eds.), Springer-Verlag, Berlin.

[7] Marko Modsching, Ronny Kramer, Klaus ten Hagen, "Field Trial on GPS Accuracy in a medium size city: The influence of built-up" in $3^{\text {rd }}$ Workshop on Positioning, Navigation and Communication 2006 (WPNC'06), Hannover, March $16^{\text {th }} 2006$

[8] Enarro (2004): http://www.enarro.com

[9] Franke, T. (2002). Integration of Group Support Tools into an Online Tourism Advising System. Workshop on User Modeling and Decision Making in Travel and Tourism Emergent Systems, Trento, April 9-10. Accessed online (September 6, 2005). Available at: http://ectrl.itc.it/home/umtts/program.htm

[10]Freytag, Tim (2003): „Städtetourismus in Heidelberg Ergebnisbericht zur Gästebefragung 2003.“, Geographisches Institut der Universität Heidelberg.

[11] Fuijtsu (2004): http://sf.us.agentcities.net/sf/

[12] Godart, Jean-Marc (2003); Beyond the Trip Planning Problem for Effective Computer-Assisted Customization, Information and Communication Technologies in Tourism 2003, Andrew Frew et al. (eds.), Springer Computer Science

[13] Goerlitz (2003): http://www.goerlitz.de/

[14] Gretzel, Ulrike; Fesenmaier, Daniel (2005): "Persuasiveness of Preference Elicitation Processes in Destination Recommendation Systems" Information and Communication Technologies in Tourism 2005, Andrew Frew et al. (eds.), Springer Computer Science.

[15] Kaufman L.; P. J. Rousseeuw (1987): "Finding Groups in Data: an Introduction to Cluster Analysis". John Wiley \& Sons, 1990

[16] Kempermann, Astrid; Borgers, Aloys; Oppewal, Harmen; Timmermans, Harry (2003): "Predicting the Duration of Theme Park Visitors' Activities: An Ordered Logit Model Using Conjoint Choice Data" Journal of Travel Research Vol.41, Sage Publications.

[17] Kempermann, Astrid; Chang-Hyeon, Joh; Timmermans, Harry (2004): "Comparing First-time and Repeat Visitors' Activity Patterns in a Tourism Environment"
Consumer Psychology of Tourism, Hospitality and Leisure Vol.3, CAB International

[18] Larson, Bradlow, Fader (2005): “An Exploratory Look at Supermarket Shopping Paths", Wharton School of the University of Pennsylvania, July 2004

[19] Lin, J. (1991): Divergence measures based on the Shannon entropy, Information Theory, IEEE Transactions on, pages $145-151$

[20] Lowry, Richard (1999-2005): "Concepts and Applications of Inferential Statistics", Subchapter 3b: Rank-Order Correlation. http://faculty.vassar.edu/lowry/webtext.html

[21] Navigon (2004): http://www.navigon.de

[22] Ricci, Francesco; Woeber, Karl; Zins, Andreas (2005): "Recommendations by Collaborative Browsing", Information and Communication Technologies in Tourism 2005 eds. Andrew Frew, Springer Computer Science (ENTER 2005)

[23] Schmidt-Belz, Barbara; Laamanen, Heimo; Poslad, Stefan; Zipf, Alexander (2003): Location-based Mobile Tourist Services - First User, Information and Communication Technologies in Tourism 2003, Andrew Frew et al. (eds.), Springer Computer Science

[24] Shoval, Noam; Isaacson, Michal (2005): "The Application of Tracking Technologies to the Study of Pedestrian Spatial Behaviour" The Professional Geographer

[25]Ten Hagen, K.; Kramer, R.; Müller, P.; Schumann, B.; Hermkes, M. (2004): Semantic Matching and Heuristic Search for a Dynamic Tour Guide, Information and Communication Technologies in Tourism 2005, A. Frew et al. (eds.), Springer Computer Science

[26] Fink, Josef and Kobsa, Alfred. User Modeling for Personalized City Tours, in Artificial Intelligence Review, vol. 18, p.33-74, Kluwer Academics Publishers

\section{Author Biographies}

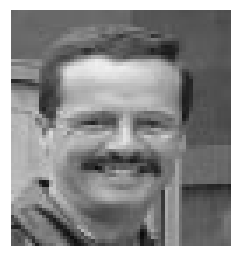

Prof. Dr.-Ing. Klaus ten Hagen studied EE/CS from 1983-1987 at the Technical University of Aachen (RWTH). He started as a developer of hardware and real-time software at the Institute for Plastics Processing (IKV). From 1988 to 1994 he was a research assistant at the Institute for Integrated Systems (ISS). His research area was hardware-software codesign. One outcome of his work was the HW/SW cosimulation capability in the system simulator COSSAP. (COSSAP was productized by CADIS. CADIS was acquired by Synopsys in 1994.) Prof. ten Hagen is the author of the book Abstract Modeling of Digital Circuits published by Springer. In 1995 he worked as a consultant for high level design methodology. From 1995 to 2000 he held various management positions at Synopsys in the Silicon Valley. From 2000-2001 he developed the patent eSilicon Access WIP tracking system and from 2001-2003 he was VP of Engineering at SigmaQuest. Since March 2003 he is a professor at the university of Zittau-Goerlitz. Prof. ten Hagen is the author of numerous publications and two US patents. More information about his 
research interests and academic functions can be found at www.ten-Hagen.org.

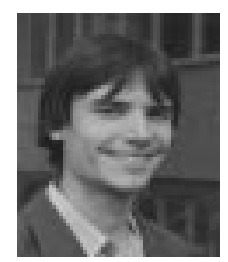

Dipl.-Inf. Ronny Kramer was born 1981 in Bad Muskau, but lived and went to school in Weisswasser (Saxony) where he did his Alevels in 1999. From 2000 until 2004 he studied computer science at the University of Applied Sciences in Zittau-Goerlitz. As his diploma he researched the matchmaking of interests in eTourism applications, which also resulted in a publication. Since November of 2004 he is working as a research assistant at this university. His current work concentrates on the VESUV project (www.vesuv-projekt.de) developing software agents for secure delegation in mobile collaborative applications.

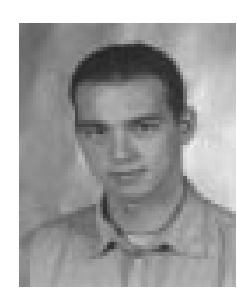

Dipl.-Inf. Marko Modsching was born 1980 in Hoyerswerda (Saxony), where he did his Alevels in 1999. After his military service in 2000 he studied computer science at the University of Applied Sciences in ZittauGoerlitz. During his study he did some research together with Prof. Dr. Joerg Schulze. There he developed a concept for a new eLearning platform called TaskTrainer. The outcome of this work was an application which is currently being used at the University of Applied Sciences Zittau/Goerlitz. As his diploma he researched for an additional TaskTrainer-module called TaskExaminator to perform softwarebased exams. Since November of 2004 he is working as a research assistant at this iniversity for the projects IKAROS (www.ikaros-projekt.de) and VESUV (www.vesuvprojekt.de). The main focus of both projects is the development of context aware software agents and services for mobile collaborative networks. 